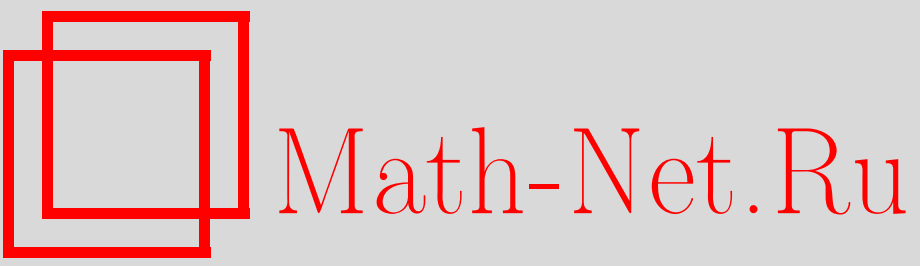

В. Г. Марихин, Представление кулоновского газа для рациональных решений уравнений Пенлеве, ТМФ, 2001, том 127, номер 2, 284-303

DOI: https://doi.org/10.4213/tmf458

Использование Общероссийского математического портала Math-Net.Ru подразумевает, что вы прочитали и согласны с пользовательским соглашением

http://www . mathnet.ru/rus/agreement

Параметры загрузки:

IP : 52.90 .164 .192

26 апреля 2023 г., 14:00:53 
ТЕОРЕТИЧЕСКАЯ

И МАТЕМАТИЧЕСКАЯ

ФИЗИКА

Том 127, № 2

май, 2001

(C) 2001 г.

В. Г. Марихин*

\title{
ПРЕДСТАВЛЕНИЕ КУЛОНОВСКОГО ГАЗА ДЛЯ РАЦИОНАЛЬНЫХ РЕШЕНИЙ УРАВНЕНИЙ ПЕНЛЕВЕ
}

\begin{abstract}
Рассматриваются рациональные решения ряда динамических систем типа нелинейного уравнения Шредингера, в частности системы Леви. Получены уравнения динамики полюсов и преобразования Беклунда для этих решений. Показано, что возможна редукция этих решений в рациональные решения уравнения Пенлеве IV, причем уравнения динамики полюсов переходят в стационарные уравнения для двумерного кулоновского газа в параболическом потенциале. Соответствующие кулоновские системы получены для уравнений Пенлеве II-VI. С помощью гамильтонова формализма построено спиновое представление для уравнений Пенлеве.
\end{abstract}

\section{1. ВВЕДЕНИЕ}

Уравнения Пенлеве I-VI [1], известные еше с конца прошлого века, в настоящее время привлекают пристальное внимание из-за их широкого применения в различных областях физики и математики. Изначально они были получены Пенлеве как дифференциальные уравнения второго порядка, решения которых не обладают подвижными особыми точками, кроме полюсов. На самом деле, подвижные особенности решений всех уравнений Пенлеве, кроме уравнения Пенлеве I, являются полюсами первого порядка. Уравнение Пенлеве І выделяется из обшего ряда из-за того, что, во-первых, его решения могут иметь особенности в виде полюсов второго порядка, а во-вторых, это уравнение, по-видимому, не является интегрируемым, поскольку не имеет преобразований Беклунда.

Как известно, некоторые уравнения Пенлеве могут быть получены как автомодельные редукции интегрируемых динамических систем. Например, в работе [2] было показано, что уравнение Пенлеве IV может быть получено как автомодельная редукция нелинейного уравнения Шредингера (НШ), а в работе [3] этот факт был обобщен на все семейство одномерных интегрируемых систем типа НШ, а кроме того, показано, что все

${ }^{*}$ Институт теоретической физики им. Л. Д. Ландау РАН, Черноголовка, Московская область, Россия. E-mail: mvg@itp.ac.ru 
рациональные решения уравнения Пенлеве IV определяют решение задачи о стационарном распределении двумерного кулоновского газа в параболическом потенциале.

В данной работе построена многочастичная система, описывающая динамику полюсов рациональных решений системы Леви - динамической системы типа НШ. Оказывается, что динамика частиц с зарядом одного знака (т.е. динамика полюсов с вычетами одного знака) эквивалентна системе Мозера. Похожая система была ранее получена (см., например, с. 234 в книге [4]) как динамика полюсов уравнения Бенджамина-Оно. Отметим, однако, что в случае системы Бенджамина-Оно полюсы рациональных решений с противоположными по знаку вычетами должны находиться в комплексно-сопряженных точках, а в случае системы Леви это ограничение отсутствует.

В работе будут получены преобразования Беклунда рассматриваемой многочастичной системы. Цепочку таких преобразований Беклунда можно рассматривать как чисто дискретную версию системы Мозера. С помошью автомодельной редукции получены все рациональные решения уравнения Пенлеве IV в виде разложения по полюсам, которые далее интерпретируются как система электрических зарядов (заряд равен вычету) с кулоновским взаимодействием в плоскости. Мы рассмотрим представление кулоновского газа для рациональных решений всех (кроме первого) уравнений Пенлеве. Для всех уравнений Пенлеве построено представление динамики магнитного момента во внешнем поле.

\section{2. ДИНАМИКА ПОЛЮСОВ РАЦИОНАЛЬНЫХ РЕШЕНИЙ СИСТЕМЫ ЛЕВИ}

Динамическая система Леви имеет вид

$$
a_{t}=\partial_{x}\left(a_{x}+a^{2}+2 a b+\nu a\right), \quad b_{t}=\partial_{x}\left(-b_{x}+2 a b+b^{2}+\nu b\right) .
$$

Используя анализ особенностей и асимптотик решений системы (1), нетрудно показать, что общее рациональное решение этой системы имеет вид

$$
a=\alpha x+\beta+\sum_{w} \frac{1}{x-w}-\sum_{v} \frac{1}{x-v}, \quad b=\alpha^{\prime} x+\beta^{\prime}+\sum_{v} \frac{1}{x-v}-\sum_{\breve{w}} \frac{1}{x-\check{w}},
$$

причем зависимость от времени величин $\alpha, \alpha^{\prime}, \beta, \beta^{\prime}$ определяется следуюшими уравнениями:

$$
\begin{array}{ll}
\alpha_{t}=2 \alpha\left(\alpha+2 \alpha^{\prime}\right), & \alpha_{t}^{\prime}=2 \alpha^{\prime}\left(\alpha^{\prime}+2 \alpha\right), \\
\beta_{t}=\beta\left(\alpha+2 \alpha^{\prime}\right)+\alpha\left(\beta+2 \beta^{\prime}+\nu\right), & \beta_{t}^{\prime}=\beta^{\prime}\left(\alpha^{\prime}+2 \alpha\right)+\alpha^{\prime}\left(\beta^{\prime}+2 \beta+\nu\right) .
\end{array}
$$

Пусть элемент $a$ принадлежит набору $\{a\}$, причем число элементов набора не фиксируется. Для упрощения записи введем следующие обозначения:

$$
\sum f(x-a)=\sum_{a \in\{a\}} f(x-a), \quad \sum f\left(a-a^{\prime}\right)=\sum_{a^{\prime} \in\{a\}, a^{\prime} \neq a} f\left(a-a^{\prime}\right) .
$$


Подставляя (2) в (1), получим систему, описывающую динамику полюсов рациональных решений системы Леви (1):

$$
\begin{aligned}
& -\frac{1}{2} w_{t}=\left(\alpha+\alpha^{\prime}\right) w+\beta+\beta^{\prime}+\frac{\nu}{2}+\sum \frac{1}{w-w^{\prime}}-\sum \frac{1}{w-\check{w}}, \\
& -\frac{1}{2} \check{w}_{t}=\left(\alpha+\alpha^{\prime}\right) \check{w}+\beta+\beta^{\prime}+\frac{\nu}{2}+\sum \frac{1}{\check{w}-w}-\sum \frac{1}{\check{w}-\check{w}^{\prime}},
\end{aligned}
$$

и соотношения на $w$ и $v$ (подробнее см. п. 2.1).

Введем новые переменные

$$
w=f \mathbf{w}+g, \quad v=f \mathbf{v}+g, \quad \check{w}=f \check{\mathbf{w}}+g,
$$

причем

$$
f_{t}=-2\left(\alpha+\alpha^{\prime}\right) f, \quad g_{t}=-2\left(\alpha+\alpha^{\prime}\right)-2\left(\beta+\beta^{\prime}\right)-\nu, \quad d \xi=-2 \frac{d t}{f^{2}} .
$$

Подставляя (4) в (3), имеем

$$
\mathbf{w}_{\xi}=\sum \frac{1}{\mathbf{w}-\mathbf{w}^{\prime}}-\sum \frac{1}{\mathbf{w}-\check{\mathbf{w}}}, \quad \check{\mathbf{w}}_{\xi}=\sum \frac{1}{\check{\mathbf{w}}-\mathbf{w}}-\sum \frac{1}{\check{\mathbf{w}}-\check{\mathbf{w}}^{\prime}}
$$

и аналогичные уравнения для $\mathbf{v}$.

Можно переписать систему (5) следуюшим образом:

$$
x_{i, \xi}=\sum_{j \neq i} \frac{q_{j}}{x_{i}-x_{j}}, \quad q_{i}^{2}=1,
$$

при этом $x_{i} \in\{\mathbf{w}\}$, если $q_{i}=1$, и $x_{i} \in\{\check{\mathbf{w}}\}$, если $q_{i}=-1$. Система (6) описывает движение электрических зарядов в плоскости, причем скорость зарядов пропорциональна действующей на них силе (динамика ланжевеновского типа).

Интересно, что можно получить уравнения, описывающие динамику только положительных или только отрицательных зарядов. Действительно, дифференцируя первое уравнение в (5) и исключая ш̌ с помощью (5), получим систему Калоджеро-Мозера (см. также с. 234 в книге [4])

$$
\mathbf{w}_{\xi \xi}=\sum \frac{1}{\left(\mathbf{w}-\mathbf{w}^{\prime}\right)^{3}}, \quad \mathbf{v}_{\xi \xi}=\sum \frac{1}{\left(\mathbf{v}-\mathbf{v}^{\prime}\right)^{3}} .
$$

Автомодельная редукция системы Леви приводит к уравнению Пенлеве IV непосредственно (без использования преобразования Миуры) [3]. Что касается рациональных решений, то автомодельная подстановка $x_{i}=\eta_{i}(2 \gamma t)^{1 / 2}$ в (6) позволяет легко получить представление кулоновского газа для рациональных решений уравнения Пенлеве IV:

$$
\gamma \eta_{i}=\sum_{j \neq i} \frac{q_{j}}{\eta_{i}-\eta_{j}}, \quad q_{i}^{2}=1
$$

Позднее мы получим представление кулоновского газа для уравнений Пенлеве II-VI, используя анализ поведения решений вблизи особых точек. 
2.1. Преобразования Беклунда для рациональных решений системы Леви. Применяя композицию преобразований Беклунда, можно получить чисто дискретную интегрируемую систему, эквивалентную данной динамической системе в том смысле, что любое решение динамической системы можно размножить, применяя преобразования Беклунда и получая тем самым решение для дискретной системы.

Цепочка (композиция) преобразований Беклунда для системы Леви есть цепочка Вольтерра. Действительно, используя преобразование

$$
a_{x}=a(\hat{b}-b), \quad \hat{b}_{x}=\hat{b}(\hat{a}-a),
$$

можно получить новое решение $(\hat{a}, \hat{b})$ системы Леви $(1)$ из решения $(a, b)$.

Пусть $a=a_{n}, \hat{a}=a_{n+1}$ (здесь и далее $x=x_{n}, \hat{x}=x_{n+1}, \check{x}=x_{n-1}$ ), тогда $a_{n, x}=$ $a_{n}\left(b_{n+1}-b_{n}\right), b_{n, x}=b_{n}\left(a_{n}-a_{n-1}\right)$. С другой стороны, обозначая $b=c_{m-1}, a=c_{m}$, $\hat{b}=c_{m+1}, \hat{a}=c_{m+2}$, нетрудно получить цепочку Вольтерра в ее обычной записи:

$$
c_{m, x}=c_{n}\left(c_{m+1}-c_{m-1}\right) .
$$

Подставляя общее рациональное решение (2) в (8), имеем

$$
\hat{\alpha}=\alpha, \quad \hat{\beta}=\beta, \quad \hat{\alpha}^{\prime}=\alpha^{\prime}, \quad \hat{\beta}^{\prime}=\beta^{\prime}, \quad N_{\hat{a}}=N_{a}+1, \quad N_{\hat{b}}=N_{b}+1,
$$

где $N_{f}$ - сумма вычетов функции $f$.

Преобразования Беклунда для рациональных решений системы (1) определяются соотношениями (они следуют из первого уравнения системы (1))

$$
\begin{aligned}
\alpha w+\beta & =\sum \frac{1}{w-\hat{v}}-2 \sum \frac{1}{w-w^{\prime}}+\sum \frac{1}{w-\check{w}} \\
\alpha v+\beta & =-\sum \frac{1}{v-\hat{v}}+2 \sum \frac{1}{v-v^{\prime}}-\sum \frac{1}{v-\check{w}} ; \\
\alpha v+\beta & =\sum \frac{1}{v-\check{v}}-\sum \frac{1}{v-\check{w}}, \quad \alpha w+\beta=\sum \frac{1}{w-\hat{v}}-\sum \frac{1}{w-\widehat{w}} .
\end{aligned}
$$

Второе уравнение системы (1) дает

$$
\begin{aligned}
\alpha^{\prime} w+\beta^{\prime} & =-\sum \frac{1}{w-\widehat{w}}+2 \sum \frac{1}{w-w^{\prime}}-\sum \frac{1}{w-v} \\
\alpha^{\prime} v+\beta^{\prime} & =\sum \frac{1}{v-w}-2 \sum \frac{1}{v-v^{\prime}}+\sum \frac{1}{v-\check{v}} \\
\alpha^{\prime} v+\beta^{\prime} & =\sum \frac{1}{v-w}-\sum \frac{1}{v-\hat{v}}, \quad \alpha^{\prime} w+\beta^{\prime}=\sum \frac{1}{w-\check{w}}-\sum \frac{1}{w-v} .
\end{aligned}
$$

Уравнения (11) и (13) можно записать в виде уравнений на полиномы:

$$
\begin{gathered}
W_{n+1}^{\prime} W_{n}-W_{n}^{\prime} W_{n+1}+\left(\alpha_{n} x+\beta_{n}\right) W_{n} W_{n+1}=\alpha_{n} W_{n+2} W_{n-1}, \\
W_{2 n}(x)=\prod_{w}\left(x-w_{n}\right), \quad W_{2 n-1}(x)=\prod_{v}\left(x-v_{n}\right), \\
\alpha_{2 n}=\alpha, \quad \alpha_{2 n-1}=\alpha^{\prime}, \quad \beta_{2 n}=\beta, \quad \beta_{2 n-1}=\beta^{\prime} .
\end{gathered}
$$


Из уравнений (10)-(13) легко получить дискретное уравнение Калоджсеро-Мозера (определяющее преобразования Беклунда системы Калоджеро-Мозера):

$$
\begin{aligned}
-\sum \frac{1}{w-\widehat{w}}+2 \sum \frac{1}{w-w^{\prime}}-\sum \frac{1}{w-\check{w}} & =0, \\
-\sum \frac{1}{v-\hat{v}}+2 \sum \frac{1}{v-v^{\prime}}-\sum \frac{1}{v-\check{v}} & =0 .
\end{aligned}
$$

Как известно (см., например, [5]), можно построить двумерную решетку преобразований Беклунда системы Леви. Для этого рассмотрим совместную с (8) систему

$$
a_{m}^{n}+b_{m}^{n}+\nu^{n}=a_{m-1}^{n+1}+b_{m}^{n+1}+\nu^{n+1}, \quad a_{m}^{n} b_{m+1}^{n}=a_{m}^{n+1} b_{m}^{n+1},
$$

где индекс $m$ определяет сдвиг вдоль цепочки Вольтерра (9), а индекс $n-$ сдвиг вдоль релятивистской цепочки Вольтерра.

Подстановка общего рационального решения (2) в (16) приводит к следующим соотношениям:

$$
\begin{gathered}
w_{m}^{n}=v_{m}^{n+1}, \quad \alpha^{n}+\alpha^{\prime n}=\alpha^{n+1}+\alpha^{\prime n+1}, \quad \alpha^{n} \alpha^{\prime n}=\alpha^{n+1} \alpha^{\prime n+1}, \\
\beta^{n}+\beta^{\prime n}+\nu^{n}=\beta^{n+1}+\beta^{\prime n+1}+\nu^{n+1}, \\
\alpha^{n} \beta^{\prime n}+\alpha^{\prime n} \beta^{n}=\alpha^{n+1} \beta^{\prime n+1}+\alpha^{\prime n+1} \beta^{n+1}, \\
\beta^{n} \beta^{\prime n}+\alpha^{n}\left(N_{b^{n}}+1\right)+\alpha^{\prime n} N_{a^{n}}=\beta^{n+1} \beta^{\prime n+1}+\alpha^{n+1} N_{b^{n+1}}+\alpha^{\prime n+1} N_{a^{n+1}} .
\end{gathered}
$$

Можно выписать также уравнения, аналогичные (14), на двумерной решетке:

$$
\begin{aligned}
& W_{m, x}^{n+1} W_{m}^{n}-W_{m}^{n+1} W_{m, x}^{n}+\left(\alpha_{n+m} x+\beta_{n+m}\right) W_{m}^{n+1} W_{m}^{n}= \\
& \quad=\alpha_{n+m} W_{m+1}^{n} W_{m-1}^{n+1}, \\
& W_{m, x}^{n} W_{m+1}^{n}-W_{m}^{n} W_{m+1, x}^{n}+\left(\alpha^{n+m} x+\beta^{n+m}\right) W_{m}^{n} W_{m+1}^{n}= \\
& \quad=\alpha^{n+m} W_{m}^{n+1} W_{m+1}^{n-1},
\end{aligned}
$$

где $W=W_{m}^{n+1}, V=W_{m}^{n}, \check{W}=w_{m+1}^{n}, \check{V}=W_{m+1}^{n-1}$

Помимо обычных симметрий, у систем типа НШ имеются и мастер-симметрии (см. [6], где мастер-симметрии выписаны в наиболее удобной форме). Например, для системы Леви мастер-симметрия имеет вид

$$
a_{\tau}=x a_{t}+a^{2}+3 a b+(\nu+2) a_{x}, \quad b_{\tau}=x b_{t}+b^{2}+3 a b+(\nu-2) b_{x}, \quad \mu=\nu+2 .
$$

Подставляя анзац (2) в (19), имеем

$$
w_{\tau}=w w_{t}-(\nu+1), \quad v_{\tau}=v v_{t}-\nu, \quad \hat{\nu}=\nu+2 .
$$

Интересно, что динамика полюсов по времени $\tau$, соответствующая мастер-симметрии (20), определяется уравнением Хопфа с ненулевой правой частью, причем решение, например, для $v$ может быть записано в неявном виде:

$$
g(v-\nu \tau)=t+\tau v-\nu \frac{\tau^{2}}{2}
$$

где $g$ - произвольная функция. 
2.2. Степени полиномов. Возможные замыкания. До сих пор мы рассматривали уравнения, описываюшие динамику полюсов рациональных решений системы Леви, а также преобразования Беклунда для них, не указывая явно допустимое число полюсов в каждом из этих решений. Нетрудно получить уравнения для степени полиномов в (17) и (18). Ответ зависит от $\alpha$ и $\alpha^{\prime}$, которые не меняются вдоль цепочки Вольтерра. Выпишем ответ для разных случаев и укажем возможное замыкание решетки.

Случай $\alpha \neq 0, \alpha^{\prime} \neq 0$. Уравнения:

$$
N_{k}^{n}+N_{k}^{n+1}+1=N_{k-1}^{n+1}+N_{k+1}^{n}, \quad N_{k}^{n}+N_{k-1}^{n+1}+1=N_{k-1}^{n}+N_{k}^{n+1} .
$$

Общее решение:

$$
N_{k}^{n}=\left(k+\frac{n+l}{2}\right)^{2}+L_{n}, \quad l \in \mathbb{Z}, \quad L_{n}+\left(\frac{n+l}{2}\right)^{2} \in \mathbb{Z} .
$$

Замыкание:

$$
N_{k+k_{0}}^{n+n_{0}}=N_{k}^{n}, \quad n_{0}=-2 k_{0}, \quad L_{n}=L_{n+2 k_{0}} .
$$

Случай $\alpha \neq 0, \alpha^{\prime}=0$. Уравнения:

$$
N_{k}^{n}+N_{k}^{n+1}+1=N_{k-1}^{n+1}+N_{k+1}^{n}, \quad N_{k}^{n}+N_{k-1}^{n+1}=N_{k-1}^{n}+N_{k}^{n+1} .
$$

Общее решение:

$$
N_{k}^{n}=\frac{1}{2} k(k-1)+k l+L_{n}, \quad l \in \mathbb{Z}, \quad L_{n} \in \mathbb{Z} .
$$

Замыкание:

$$
N_{k+k_{0}}^{n+n_{0}}=N_{k}^{n}, \quad k_{0}=0, \quad L_{n}=L_{n+n_{0}} .
$$

2.3. Система Тоды. Применяя преобразование Миуры

$$
B=a+b, \quad A=a \hat{b}=a b+a_{x},
$$

получим динамическую систему Тоды

$$
A_{t}=\partial_{x} \frac{\delta H}{\delta B}, \quad B_{t}=\partial_{x} \frac{\delta H}{\delta A}, \quad H=A_{x} B+A B^{2}+A^{2} .
$$

Дуальное преобразование Миуры имеет вид

$$
\widetilde{B}=a+\hat{b}=a+b+\frac{a_{x}}{a}, \quad \tilde{A}=a b,
$$

дуальная к (21) система -

$$
\tilde{A}_{t}=\partial_{x} \frac{\delta \widetilde{H}}{\delta \widetilde{B}}, \quad \widetilde{B}_{t}=\partial_{x} \frac{\delta \widetilde{H}}{\delta \tilde{A}}, \quad \widetilde{H}=-\tilde{A}_{x} \widetilde{B}+\tilde{A} \widetilde{B}^{2}+\tilde{A}^{2} .
$$


Отметим, что пара $(\tilde{A}, \check{\widetilde{B}})$ удовлетворяет системе $(21)$. Рациональные решения систем (21) и (22) можно записать в виде

$$
\begin{aligned}
& B(x, t)=\left(\alpha+\alpha^{\prime}\right) x+\beta+\beta^{\prime}+\sum \frac{1}{x-w}-\sum \frac{1}{x-\breve{w}}, \\
& \widetilde{B}(x, t)=\left(\alpha+\alpha^{\prime}\right) x+\beta+\beta^{\prime}+\sum \frac{1}{x-\hat{v}}-\sum \frac{1}{x-v} .
\end{aligned}
$$

Учитывая уравнения (10)-(13) и (15), получим

$$
\begin{aligned}
& A=(\alpha x+\beta)\left(\alpha^{\prime} x+\beta^{\prime}\right)+\alpha\left(N_{b}+1\right)+\alpha^{\prime} N_{a}-\sum \frac{1}{(x-w)^{2}}, \\
& \tilde{A}=(\alpha x+\beta)\left(\alpha^{\prime} x+\beta^{\prime}\right)+\alpha N_{b}+\alpha^{\prime} N_{a}-\sum \frac{1}{(x-v)^{2}} .
\end{aligned}
$$

Нетрудно убедиться, что величины $p=\tilde{A}, z=\nu+\check{\widetilde{B}}$ удовлетворяют $(p, z)$-версии цепочки Тоды

$$
p_{x}=p(\hat{z}-z), \quad z_{x}=p-\check{p} .
$$

Перейдем теперь к двумерному случаю. Рассмотрим одну из систем Дэви-Стюартсона

$$
u_{t}=u_{x x}+2(u V)_{x}, \quad v_{t}=-v_{x x}+\left(V^{2}\right)_{y}+2 u_{x}, \quad V_{y}=v_{x} .
$$

Эта система является гамильтоновой, причем

$$
v=q_{y}, \quad u=p, \quad H=p_{x} q_{x}+p q_{x}^{2}+p \partial_{y}^{-1} \partial_{x} p
$$

Отметим, что редукция гамильтониана (25) к одномерному случаю $\partial_{y}^{-1} \partial_{x} \rightarrow 1$ приводит к гамильтониану системы Тоды (21), если $A=p, B=q_{x}$. Используя соотношения (23), ишем рациональные решения системы (24) в виде

$$
q=\ln \frac{W_{n}}{W_{n-1}}, \quad p=\frac{W_{n-1} W_{n+1}}{W_{n}^{2}}, \quad W_{n}=Q_{2 n} .
$$

Тогда полюсы $w_{n}(y, t)$ удовлетворяют системе Калоджеро-Мозера $(7)$ по $t$, как и в одномерном случае. Отметим, что для уравнений Кадомцева-Петвиашвили и Кортевега-де Фриза ситуация несколько сложнее, поскольку из результатов Мозера и теоремы Кричевера (см., например, [7]) следует, что динамика полюсов по $t$ в этом случае определяется гамильтонианом $H_{3}$, а не более простым $H_{2}$, как в случае систем Леви и Дэви-Стюартсона.

Что же касается динамики по $y$, то имеет место соотношение

$$
w_{n, y}=\frac{W_{n-1}\left(w_{n}\right) W_{n+1}\left(w_{n}\right)}{W_{n}^{\prime 2}\left(w_{n}\right)}, \quad W_{n}\left(w_{n}\right)=0 .
$$




\section{3. ПРЕДСТАВЛЕНИЕ КУЛОНОВСКОГО ГАЗА И ПРЕОБРАЗОВАНИЯ БЕКЛУНДА УРАВНЕНИЙ ПЕНЛЕВЕ}

В современной литературе принято рассматривать следующие шесть уравнений Пенлеве $(a, b, c, d$ - константы):

$$
\begin{aligned}
w_{x x}= & 6 w^{2}+x \\
w_{x x}= & 2 w^{3}+x w+a \\
w_{x x}= & \frac{w_{x}^{2}}{w}-\frac{w_{x}}{x}+\frac{a w^{2}+b}{x}+c w^{3}+\frac{d}{w} \\
w_{x x}= & \frac{w_{x}^{2}}{2 w}+\frac{3 w^{3}}{2}+4 x w^{2}+2\left(x^{2}-a\right) w+\frac{b}{w}, \\
w_{x x}= & \left(\frac{1}{2 w}+\frac{1}{w-1}\right) w_{x}^{2}-\frac{w_{x}}{x}+\frac{2}{x^{2}}(w-1)^{2}\left(a w+\frac{b}{w}\right)+ \\
& +\frac{c w}{x}+\frac{d w(w+1)}{w-1}, \\
w_{x x}= & \frac{1}{2}\left(\frac{1}{w}+\frac{1}{w-1}+\frac{1}{w-x}\right) w_{x}^{2}-\left(\frac{1}{x}+\frac{1}{x-1}+\frac{1}{w-x}\right) w_{x}+ \\
& +\frac{w(w-1)(w-x)}{x^{2}(x-1)^{2}}\left[a+\frac{b x}{w^{2}}+\frac{c}{(w-1)^{2}}(x-1)+\frac{d x(x-1)}{(w-x)^{2}}\right] .
\end{aligned}
$$

Все уравнения Пенлеве, кроме первого (т.е. (PII)-(PVI)), могут иметь подвижные особые точки в виде простых полюсов, причем решение вблизи этих особых точек ведет себя следуюшим образом:

$$
w(x)=\frac{q}{x-z}+g+o(x-z), \quad x \rightarrow z,
$$

заряд $q$ (вычет) не зависит от положения полюса для уравнений (PII)-(PIV), причем $q^{2}=$ const $\left(q^{2}=1\right.$ для уравнений (PII) и (PIV)). Коэффициент $g$ для уравнений (PII)(PIV) однозначно связан с положением полюса $z$ (для уравнений (PV) и (PVI) это не так), и применима следуюшая процедура построения уравнений кулоновского газа: общее рациональное решение уравнений Пенлеве имеет вид

$$
w(x)=\alpha x+\beta+\sum_{i} \frac{q_{i}}{x-x_{i}},
$$

и, используя (26), имеем

$$
g\left(x_{i}\right)-\alpha x_{i}-\beta=\sum_{j \neq i} \frac{q_{j}}{x_{i}-x_{j}} \forall i
$$

Поясним смысл термина “кулоновский газ”. Определим потенциал

$$
U=\sum_{i} q_{i}\left(G\left(x_{i}\right)-\frac{1}{2} \alpha x_{i}^{2}-\beta x_{i}\right)
$$


Тогда, варьируя функционал энергии заряженных частищ, взаимодействующих по двумерному закону Кулона и находяшихся во внешнем поле,

$$
E=U+\sum_{i>j} q_{i} q_{j} \ln \left(x_{i}-x_{j}\right),
$$

получаем систему уравнений (27). Ниже мы приведем конкретные примеры.

Все уравнения Пенлеве являются лагранжевыми, т.е. могут быть получены варьированием следующих лагранжианов:

$$
\begin{aligned}
L & =\frac{w_{x}^{2}}{2}+2 w^{3}+x w \\
L & =w_{x}^{2}+w^{4}+x w^{2}+2 a w \\
L & =\frac{w_{x}^{2}}{2 w^{2}} x+a w-\frac{b}{w}+\frac{x}{2}\left(c w^{2}-\frac{d}{w^{2}}\right) \\
L & =\frac{w_{x}^{2}}{w}+w^{3}+4 w^{2} x+4 w\left(x^{2}-a\right)-\frac{2 b}{w} \\
L & =\frac{w_{x}^{2}}{w(w-1)^{2}} x+\frac{4}{x}\left(a w-\frac{b}{w}\right)-\frac{2 c}{w-1}-\frac{2 d w}{(w-1)^{2}} x, \\
L & =\frac{w_{x}^{2}}{2} \frac{x(x-1)}{w(w-1)(w-x)}+\frac{a w}{x(x-1)}-\frac{b}{w(x-1)}-\frac{c}{x(w-1)}-\frac{d}{w-x} .
\end{aligned}
$$

Все лагранжианы уравнений Пенлеве можно привести к виду

$$
L=\frac{1}{A(w, x)}\left(w_{x}+B(w, x)\right)^{2}-4 C(w, x),
$$

поэтому удобно ввести гамильтоново описание этих систем

$$
L=p w_{x}+A(w, x) p^{2}+B(w, x) p+C(w, x)
$$

Действительно, исключая $p$ из (29), получим (28). Гамильтонова форма описания уравнений Пенлеве была введена в работе [8]. Это описание очень удобно для получения преобразований Беклунда для уравнений Пенлеве (кроме первого) в универсальной форме

$$
-\frac{1}{2 A}\left(w_{x}+B\right)=p=\frac{D}{\widehat{w}-w},
$$

причем коэффициенты гамильтониана имеют вид

$$
A=a_{3} w^{3}+a_{2} w^{2}+a_{1} w+a_{0}, \quad B=b_{2} w^{2}+b_{1} w+b_{0}, \quad C=c_{1} w .
$$

Преобразования Беклунда действуют на них следующим образом:

$$
\begin{gathered}
\hat{a}_{i}=a_{i}, \quad \hat{b}_{2}=b^{2}-3 a_{3} D, \quad \hat{b}_{1}=b_{1}-2 a_{2} D, \quad \hat{b}_{0}=b_{0}-a_{1} D, \\
c_{1}=b_{2} D-a_{3} D^{2}, \quad \hat{c}_{1}=2 a_{3} D^{2}-b_{2} D .
\end{gathered}
$$


Используя (29) и (30), можно получить динамические спиновые системы, эквивалентные уравнениям Пенлеве (PII)-(PVI):

$$
\frac{i}{2} \nu_{\mathrm{J}}(x) \vec{S}_{x}=\left[\vec{S} \times \frac{\delta H_{\mathrm{J}}}{\delta \vec{S}}\right], \quad H_{\mathrm{J}}=\left(\vec{S} \hat{I}_{\mathrm{J}} \vec{S}\right)+\left(\vec{h}_{\mathrm{J}} \vec{S}\right), \quad \vec{S}^{2}=D^{2},
$$

где $\mathrm{J}=\mathrm{II}, \ldots, \mathrm{VI}$,

$$
\nu_{\mathrm{II}}=\nu_{\mathrm{IV}}=1, \quad \nu_{\mathrm{III}}=\nu_{\mathrm{V}}=x, \quad \nu_{\mathrm{VI}}=x(x-1),
$$

а вектор спина $\vec{S}$ и гамильтонова пара $(p, w)$ связаны соотношением

$$
\vec{S}=\vec{M}(w) p+\frac{D}{2} \frac{\partial \vec{M}(w)}{w}, \quad \vec{M}(w)=\left(w^{2}-1, i w^{2}+i, 2 w\right) .
$$

Нетрудно найти тензор анизотропии $\hat{I}_{\mathrm{J}}$ и магнитное поле $\vec{h}_{\mathrm{J}}$, входящие в гамильтониан $H_{\mathrm{J}}$ в скалярной форме:

$$
\begin{aligned}
& r(w, x)=(\vec{M}(w) \hat{I}(x) \vec{M}(w))=\nu(x) A(w, x) \\
& h(w, x)=(\vec{M}(w) \vec{h}(x))=\nu(x)\left[B(w, x)-\frac{D}{2} A_{w}(w, x)\right] .
\end{aligned}
$$

Интересно отметить, что гамильтониан $H_{\mathrm{J}}$ описывает динамику спина величины $D$ в поле анизотропии $\hat{I}$ и в магнитном поле $\vec{h}$, причем произвольную систему вида $(29)$ можно привести к виду (32) в том случае, если

$$
C(w, x)=\frac{D}{2}\left[B_{w}-\frac{D}{3} A_{w w}\right]
$$

т.е. должны быть выполнены условия интегрируемости (31).

Рассмотрим теперь представление кулоновского газа и спиновой динамики для каждого из уравнений Пенлеве (PII)-(PVI).

3.1. Уравнение Пенлеве II. Лагранжиан уравнения (PII) в гамильтоновых переменных:

$$
L_{\mathrm{II}}=p w_{x}+p^{2}+p\left(w^{2}+\frac{x}{2}\right)-\frac{w}{4}(2 a+1) .
$$

Преобразования Беклунда для уравнения (PII):

$$
\widehat{w}=-w+\frac{2 a+1}{4 p}, \quad p=-\frac{1}{2}\left(w_{x}+w^{2}+\frac{x}{2}\right)=-\frac{1}{2}\left(-\widehat{w}_{x}+\widehat{w}^{2}+\frac{x}{2}\right), \quad \hat{a}=a+1 .
$$

Цепочка преобразований Беклунда:

$$
w_{n}^{2}+\frac{x}{2}+\frac{2 n-1+2 a_{0}}{w_{n-1}+w_{n}}+\frac{2 n+1+2 a_{0}}{w_{n}+w_{n+1}}=0 .
$$

Лагранжиан цепочки:

$$
L=\frac{1}{3} w_{n}^{3}+\frac{1}{2} x w_{n}+z_{n} \ln \left(w_{n}+w_{n+1}\right), \quad z_{n}=2 n+1+2 a_{0} .
$$


Рациональные решения уравнения Пенлеве II. Поведение вблизи особенностей:

$$
\begin{aligned}
w & \sim \frac{q}{x-x_{0}}-\frac{q x_{0}}{6}\left(x-x_{0}\right), & & x \rightarrow x_{0}, \quad q^{2}=1 ; \\
w & \sim-\frac{a}{x}, & & x \rightarrow \infty .
\end{aligned}
$$

Обшее рациональное решение $a \in \mathbb{Z}$ и представление кулоновского газа:

$$
w=\sum_{i} \frac{q_{i}}{x-x_{i}}, \quad q_{i}= \pm 1, \quad \sum_{i} q_{i}=-a, \quad \sum_{j \neq i} \frac{q_{j}}{x_{i}-x_{j}}=0 \quad \forall i
$$

Примеры рациональных решений $(w=w(x, a))$ :

$$
w(x, 0)=0, \quad w(x, \pm 1)=\mp \frac{1}{x}, \quad w(x, \pm 2)=\mp \frac{2}{x} \frac{x^{3}-2}{x^{3}+4} .
$$

Например, решение

$$
w(x, 2)=\frac{1}{x}-\frac{1}{x-x_{1}}-\frac{1}{x-x_{2}}-\frac{1}{x-x_{3}}, \quad x_{k}^{3}=-4, \quad k=1,2,3,
$$

соответствует конфигурации зарядов, при которой один положительный заряд помешен в начало координат $x=0$, а три отрицательных - в вершины равностороннего треугольника с координатами $x=x_{k}$, причем внешний потенциал отсутствует.

Поле анизотропии и магнитное поле для спинового представления (32), (33) уравнения Пенлеве II имеют вид

$$
r=1, \quad h=w^{2}+\frac{x}{2}, \quad D=-\frac{2 a+1}{4} .
$$

3.2. Уравнение Пенлеве III. Лагранжиан уравнения (РIII) в гамильтоновых переменных имеет вид

$$
L_{\mathrm{III}}=p w_{x}+\frac{1}{x} p^{2} w^{2}+p\left(A w^{2}+\frac{B}{x} w+C\right)+A D w
$$

причем

$$
a=A(B-2 D-1), \quad b=-C(B+1), \quad c=A^{2}, \quad d=-C^{2} .
$$

Преобразования Беклунда:

$$
\widehat{w}=w+\frac{D}{p}, \quad p=-\frac{x}{2 w^{2}}\left(w_{x}+A w^{2}+\frac{B}{x} w+C\right) .
$$

Преобразование калибровочных параметров:

$$
\hat{A}=A, \quad \widehat{B}=B-2 D, \quad \widehat{C}=C, \quad \widehat{D}=-D .
$$


Параметры уравнения (PIII) выражаются через калибровочные параметры следуюшим образом:

$$
\hat{a}=A(B-1), \quad \hat{b}=-C(B+1)+2 C D, \quad \hat{c}=c, \quad \hat{d}=d .
$$

Цепочка преобразований Беклунда:

$$
-\frac{1}{\widehat{w}-w}+\frac{1}{w-\check{w}}=\frac{1}{D}\left(A x+x \frac{C}{w^{2}}-\frac{b}{C w}\right) .
$$

Лагранжиан цепочки:

$$
L=D \ln \left(w_{n+1}-w_{n}\right)-A x w_{n}+C \frac{x}{w_{n}}+\frac{b_{n}}{C} \ln w_{n} .
$$

Рациональные решения уравнения Пенлеве III. Поведение вблизи особенностей:

$$
\begin{aligned}
w & \sim \frac{q}{x-x_{0}}-\frac{q+\frac{a}{c}}{2 x_{0}}, \quad x \rightarrow x_{0}, \quad q^{2}=\frac{1}{c} \\
w & \sim w(\infty)=A, \quad x \rightarrow \infty, \quad A^{4}=-\frac{d}{c} .
\end{aligned}
$$

Общее рациональное решение $a \in \mathbb{Z}$ и представление кулоновского газа:

$$
w=A+\sum_{i} \frac{q_{i}}{x-x_{i}}, \quad q_{i}^{2}=\frac{1}{c}, \quad \sum_{j \neq i} \frac{q_{j}}{x_{i}-x_{j}}=-A-\frac{q_{i}+\frac{a}{c}}{2 x_{i}} \quad \forall i .
$$

Поле анизотропии и магнитное поле для спинового представления $(32),(33)$ имеют вид

$$
r=w^{2}, \quad h=A x w^{2}+(B-D) w+C x .
$$

3.3. Уравнение Пенлеве IV. Лагранжиан уравнения (PIV) в гамильтоновых переменных имеет вид

$$
L_{\mathrm{IV}}=p w_{x}+p^{2} w+p\left(w^{2}+2 w x+A\right)+B w, \quad a=1+B-\frac{1}{2} A, \quad b=-\frac{1}{2} A^{2} .
$$

Преобразования Беклунда:

$$
\widehat{w}=w+\frac{B}{p}, \quad p=-\frac{1}{2 w}\left(w_{x}+w^{2}+2 w x+A\right), \quad \hat{A}=A-B, \quad \widehat{B}=-B .
$$

Калибровочное преобразование параметров:

$$
\hat{p}=p+\frac{A}{w}, \quad \hat{A}=-A, \quad \widehat{B}=B-A .
$$


Лагранжиан уравнения (PIV) также может иметь вид

$$
L_{\mathrm{IV}}=p w_{x}+p^{2} w-p\left(w^{2}+2 w x+\tilde{A}\right)+\widetilde{B} w, \quad a=-1+\widetilde{B}-\frac{1}{2} \tilde{A}, \quad b=-\frac{1}{2} \tilde{A}^{2} .
$$

Преобразования Беклунда:

$$
\widehat{w}=w-\frac{\widetilde{B}}{p}, \quad p=\frac{1}{2 w}\left(-w_{x}+w^{2}+2 w x+\tilde{A}\right), \quad \hat{\tilde{A}}=\tilde{A}-\widetilde{B}, \quad \widehat{\widetilde{B}}=-\widetilde{B} .
$$

Калибровочное преобразование параметров:

$$
\hat{p}=p-\frac{\tilde{A}}{w}, \quad \hat{\tilde{A}}=-\tilde{A}, \quad \widehat{\widetilde{B}}=\widetilde{B}-\tilde{A} .
$$

Цепочка преобразований Беклунда:

$$
\widehat{w}+\check{w}=-\left(w+2 A x \frac{1}{w}\right) .
$$

Лагранжиан цепочки:

$$
L=w_{n} w_{n+1}+\frac{1}{2} w_{n}^{2}+2 x w_{n}+A_{n} \ln w_{n}
$$

Уравнение Пенлеве IV имеет дополнительную симметрию третьего порядка [9]. Действительно, лагранжиан (LIV) может быть представлен в одной из трех эквивалентных форм $(u+v+s=-2 x)$ :

$$
\begin{aligned}
L & =u v^{\prime}-u v s+c_{1} u+c_{2} v=s u^{\prime}-u v s-c_{2} s+\left(c_{1}-c_{2}-2\right) u= \\
& =v s^{\prime}-u v s+\left(c_{2}-c_{1}+2\right) v-c_{1} s .
\end{aligned}
$$

Исключая последовательно $u, v$ и $s$ из (34) и обозначая произвольное решение уравнения Пенлеве IV как $w(x ; a, b)$, имеем

$$
\begin{gathered}
v=w\left(x ; 1+c_{2}-\frac{1}{2} c_{1},-\frac{1}{2} c_{1}^{2}\right), \quad u=w\left(x ;-1+c_{1}-\frac{1}{2} c_{2},-\frac{1}{2} c_{2}^{2}\right), \\
s=w\left(x ;-\frac{1}{2}\left(c_{1}+c_{2}\right),-\frac{1}{2}\left(2+c_{2}-c_{1}\right)^{2}\right) .
\end{gathered}
$$

Преобразования Беклунда имеют следуюший вид:

$$
\begin{aligned}
T_{1}^{ \pm}: \mathbf{v} \rightarrow \mathbf{u} \rightarrow \mathbf{s} \rightarrow \mathbf{v} & \\
\widehat{w} & =-\frac{1}{2 w}\left(w^{\prime}+w^{2}+2 x w \pm \sqrt{-2 b}\right) \\
(a, b) \rightarrow(\hat{a}, \hat{b}) & =\left(-\frac{1}{2}(a+1) \pm \frac{3}{4} \sqrt{-2 b},-\frac{1}{2}\left(a-1 \pm \frac{1}{2} \sqrt{-2 b}\right)^{2}\right) ; \\
T_{2}^{ \pm}: \mathbf{s} \rightarrow \mathbf{u} \rightarrow \mathbf{v} \rightarrow \mathbf{s}, & \widehat{w}=-\frac{1}{2 w}\left(-w^{\prime}+w^{2}+2 x w \pm \sqrt{-2 b}\right) \\
(a, b) \rightarrow(\hat{a}, \hat{b}) & =\left(-\frac{1}{2}(a-1) \pm \frac{3}{4} \sqrt{-2 b},-\frac{1}{2}\left(a+1 \pm \frac{1}{2} \sqrt{-2 b}\right)^{2}\right) .
\end{aligned}
$$

Комбинируя преобразования (35), (36), можно построить решетку для решений уравнения (PIV). 
3.4. Решетка для уравнения (PIV). Решетка для решений уравнения (PIV) состоит из набора $q_{i j k}$, причем

$$
(i, j, k)=(i+1, j+1, k+1), \quad(i, j, k) \neq(2 l, 2 m, 2 n),
$$

т.е. она является правильной треугольной решеткой, из которой исключена подрешетка с удвоенной элементарной ячейкой. При этом имеют место следуюшие соотношения:

1) каждая прямая является цепочкой Вольтерра:

$$
q_{n}^{\prime}=-q_{n}\left(q_{n+1}-q_{n-1}\right),
$$

где $q_{n}=q_{n j k}$, или $q_{n}=q_{i n k}$, или $q_{n}=q_{i j n} ;$

2) на любом элементарном треугольнике

$$
\sum q_{\mathrm{tr}}=-2 x
$$

где суммирование проводится по вершинам треугольника.

Рациональные решения уравнения Пенлеве IV. Поведение вблизи особенностей:

$$
\begin{aligned}
w & \sim \frac{q}{x-x_{0}}-x_{0}, \quad q^{2}=1, \quad x \rightarrow x_{0} ; \\
w & \sim B x, \quad B(B+2)\left(B+\frac{2}{3}\right)=0, \quad x \rightarrow \infty ; \\
w & \sim \frac{q}{x}-\frac{2}{3}(2-a q) x, \quad q^{3}=q, \quad x \rightarrow 0 .
\end{aligned}
$$

Общее рациональное решение $a \in \mathbb{Z}$ и представление кулоновского газа:

$$
w=B x+\sum_{i} \frac{q_{i}}{x-x_{i}}, \quad q_{i}= \pm 1, \quad \sum_{j \neq i} \frac{q_{j}}{x_{i}-x_{j}}=-(1+B) x_{i} \quad \forall i .
$$

Отметим, что

$$
f(x)=\sum_{i} \frac{q_{i}}{x-x_{i}} \quad \forall i \Longleftrightarrow f^{2}-2 \mu x f+2 \mu Q=\sum_{i} \frac{q_{i}^{2}}{\left(x-x_{i}\right)^{2}}, \quad Q=\sum_{i} q_{i} .
$$

Поле анизотропии и магнитное поле для спинового представления $(32),(33)$ имеют вид

$$
r=w, \quad h=w^{2}+2 w x+A-\frac{D}{2} .
$$


3.5. Уравнение Пенлеве V. Лагранжиан уравнения (PV) в гамильтоновых переменных имеет вид

$$
L_{\mathrm{V}}=p w_{x}+\frac{1}{x} p^{2} w(w-1)^{2}+p\left(\frac{1}{x}((A+2 D) w+B)(w-1)+C w\right)+\frac{1}{x} w D(A+D)
$$

причем

$$
A^{2}=4 a, \quad B^{2}=-4 b, \quad C^{2}=-2 d, \quad-2 c=2 C(A+B+2 D+1) .
$$

Преобразования Беклунда:

$$
p=\frac{D}{\widehat{w}-w}, \quad \hat{A}=A+D, \quad \widehat{B}=B+D, \quad \widehat{C}=C, \quad \widehat{D}=-D
$$

Для уравнения (PV) можно определить несколько калибровочных преобразований параметров:

1) $\hat{A}=-A, \widehat{D}=A+D$;

2) $\widehat{B}=-B, \widehat{D}=B+D$;

3) $\widehat{C}=-C, \widehat{D}=-(A+B+D+1)$.

Цепочка имеет вид

$$
\frac{\mu}{\hat{q}-q}+\frac{\mu}{\check{q}-q}=2 \gamma x+\frac{\beta}{q-1}-\frac{\alpha}{q} .
$$

Лагранжиан цепочки

$$
L=\mu \ln \left(q_{n+1}-q_{n}\right)-2 x \gamma_{n} q_{n}-\beta_{n} \ln \left(q_{n}-1\right)+\alpha_{n} \ln q_{n},
$$

при этом

$\gamma_{n}=(-1)^{n} \gamma, \quad \alpha_{n}=\alpha_{0}+\left(\alpha_{0}+\beta_{0}\right)\left((-1)^{n}-1\right)+\frac{n}{2}, \quad \beta_{n}=\beta_{0}+\left(\alpha_{0}+\beta_{0}\right)\left((-1)^{n}-1\right)-\frac{n}{2}$.

Рациональные решения уравнения Пенлеве V. Поведение вблизи полюса $x \rightarrow z$ :

$$
\begin{gathered}
w \sim \frac{q z}{x-z}+g+h(x-z), \quad q^{2}=\frac{1}{4 a}, \\
1+4 b+2 z c+8 q+12 a+2 z^{2} d+24 g^{2} a-12 z h q-12 g q-32 g a=0 .
\end{gathered}
$$

Асимптотика $x \rightarrow \infty$ :

$$
w \sim B x, \quad B\left(d+2 a B^{2}\right)=0, \text { или } \quad w \sim \frac{D}{x}, \quad D^{2}=2 \frac{b}{d}, \text { или } \quad w \sim-1 .
$$

Поведение при $x \rightarrow 0$ :

$$
w \sim B+C x, \quad\left(B^{2}+\frac{b}{a}\right)=0, \quad C=\frac{B c}{1+2 b+8 a B-4 a},
$$


или

$$
w \sim 1+c x, \quad B=1
$$

Для уравнения Пенлеве V нельзя получить представление кулоновского газа (27), так как параметры $z$ и $g$ независимы. Однако можно получить систему уравнений типа уравнений Дубровина:

$$
\begin{array}{ll}
\prod_{j}\left(x_{i}-y_{j}\right)=q_{i} x_{i} \prod_{j \neq i}\left(x_{i}-x_{j}\right), & q_{i}^{2}=\frac{1}{4 a} \\
\prod_{j}\left(y_{i}-x_{j}\right)=Q_{i} y_{i} \prod_{j \neq i}\left(y_{i}-y_{j}\right), & Q_{i}^{2}=-\frac{1}{4 b} \quad \forall i
\end{array}
$$

где $x_{i}$ и $y_{i}$ - соответственно полюсы и нули функции $w$.

Поле анизотропии и магнитное поле для спинового представления (32), (33) имеют вид

$$
r=w(w-1)^{2}, \quad h=(w-1)\left(\left(A+\frac{D}{2}\right) w+B+\frac{D}{2}\right)+C w x .
$$

3.6. Уравнение Пенлеве VI. Точечные преобразования лагранжиана (LVI):

$$
\begin{gathered}
L\left(\frac{1}{w}, \frac{1}{x} ; a, b, c, d\right)=L(w, x ;-b,-a, c, d,), \\
L(1-w, 1-x ; a, b, c, d)=L(w, x ; a,-c,-b, d,),
\end{gathered}
$$

а также

$$
L\left(\frac{w}{x}, \frac{1}{x} ; a, b, c, d\right)=L\left(w, x ; a, b, \frac{1}{2}-d, \frac{1}{2}-c\right) .
$$

Лагранжиан уравнения (PVI) в гамильтоновых переменных имеет вид

$$
\begin{aligned}
L_{\mathrm{VI}}= & w_{x} p-\frac{1}{x(x-1)}\left[p^{2} w(w-1)(w-x)+p(A(w-1)(w-x)+B w(w-x)+\right. \\
& +(C+1) w(w-1))+D(A+B+C+1-D) w]
\end{aligned}
$$

причем

$$
a=\frac{1}{2}(A+B+C+1-2 D)^{2}, \quad b=-\frac{1}{2} A^{2}, \quad c=\frac{1}{2} B^{2}, \quad d=\frac{1}{2}\left(1-C^{2}\right) .
$$

Калибровочные преобразования:

$$
\begin{aligned}
& G_{a}: \quad L(w, p ; A, B, C, D)=L(w, \hat{p} ;-A, B, C, D-A), \quad \hat{p}=p+\frac{A}{w}, \\
& G_{b}: L(w, p ; A, B, C, D)=L(w, \hat{p} ; A,-B, C, D-B), \quad \hat{p}=p+\frac{B}{w-1}, \\
& G_{c}: \quad L(w, p ; A, B, C, D)=L(w, \hat{p} ; A, B,-C, D-C), \quad \hat{p}=p+\frac{C+1}{w-x} \text {. }
\end{aligned}
$$


Чистая калибровка:

$$
G: \quad L(w, p ; A, B, C, D)=L(w, p ; A, B, C, A+B+C+1-D) .
$$

Преобразование Беклунда:

$$
\widehat{w}-w=\frac{D}{p}, \quad S: \quad L(w, p ; A, B, C, D)=L(\widehat{w}, p ; A-D, B-D, C-D,-D),
$$

при этом

$$
p=\frac{x(x-1) w_{x}}{2 w(w-1)(w-x)}-\frac{1}{2}\left(\frac{A}{w}+\frac{B}{w-1}+\frac{C+1}{w-x}\right) .
$$

Генераторы группы преобразований (37) обладают следующими свойствами:

$$
\begin{gathered}
G_{i}^{2}=G^{2}=S^{2}=1, \quad G_{i} G_{j}=G_{j} G_{i}, \quad G_{i} G=G G_{i}, \\
\left(S G_{i}\right)^{3}=(S G)^{3}=1, \quad\left(S G G_{i}\right)^{4}=1, \\
\left(S G G_{a} G_{b} G_{c}\right)^{2}[A, B, C, D]=[A+1, B+1, C+1, D+2], \\
\left(G_{c} S G G_{a} G_{b} S G G_{a} G_{b} S\right)[A, B, C, D]=[A, B, C+2, D+1] .
\end{gathered}
$$

Рациональные решения уравнения Пенлеве VI. Поведение решения вблизи полюса $x \rightarrow z$ :

$$
\begin{gathered}
w \sim z(z-1) \frac{q}{x-z}+g+h(x-z), \quad q^{2}=\frac{1}{2 a}, \\
12 g^{2} a-8 g a z+12 g a q-24 g a q z-8 g a+2 d z^{2}-12 q h z^{2} a+2 a z^{2}+ \\
+6 z^{2}+12 q z^{2} a-6 z+2 b z+2 c z-2 d z+1+12 q h z a+2 a z+2 a-4 q a-2=0 .
\end{gathered}
$$

Поведение на бесконечности $x \rightarrow \infty$ :

$$
w \sim B x, \quad B^{2}\left((B-1)^{2}+\frac{2 d-1}{2 a}\right)=0,
$$

или

$$
w \sim C, \quad\left(C-\frac{b}{b+c}\right)^{2}+\frac{b c}{(b+c)^{2}} .
$$

Для уравнения (PVI), как и для уравнения (PV), нельзя получить представление кулоновского газа (27), так как параметры $z$ и $g$ независимы.

Выпишем систему уравнений типа уравнений Дубровина:

$$
\begin{array}{ll}
\prod_{j}\left(x_{i}-y_{j}\right)=q_{i} x_{i}\left(x_{i}-1\right) \prod_{j \neq i}\left(x_{i}-x_{j}\right), & q_{i}^{2}=\frac{1}{2 a} \\
\prod_{j}\left(y_{i}-x_{j}\right)=Q_{i} y_{i}\left(y_{i}-1\right) \prod_{j \neq i}\left(y_{i}-y_{j}\right), & Q_{i}^{2}=-\frac{1}{2 b} \quad \forall i
\end{array}
$$

где $x_{i}$ и $y_{i}$ - соответственно полюсы и нули $w$. 
Преобразование (38) действует на особенности следующим образом:

$$
\hat{q}_{+}=\frac{1}{\hat{A}+\widehat{B}+\widehat{C}+1-2 \widehat{D}}, \quad \hat{q}_{-}=0, \quad q_{ \pm}= \pm \frac{1}{A+B+C+1-2 D} .
$$

Пусть $w=w(a, b, c, d ; x)$. Приведем несколько примеров частных (рациональных) решений уравнения (PVI):

$$
\begin{gathered}
w\left(a, b, c, \frac{1}{2} ; x\right)=x ; \quad w(a, b, 0, d)=1 \\
w\left(\frac{1}{2},-\frac{1}{2} q^{2}, \frac{1}{2} q^{2}, \frac{1}{2}-2 q^{2} ; x\right)=\frac{x}{2 x-1} ; \\
w\left(2 q^{2},-\frac{1}{2}(1+q)^{2}, \frac{1}{2}(1+q)^{2}, 0\right)=\frac{x}{q} \frac{x(1+2 q)-(1+q)}{2 x-1} .
\end{gathered}
$$

Поле анизотропии и магнитное поле для спинового представления $(32),(33)$ имеют Вид

$$
\begin{gathered}
r=w(w-1)(w-x), \\
h=\left(A-\frac{D}{2}\right)(w-1)(w-x)+\left(B-\frac{D}{2}\right) w(w-x)+\left(C+1-\frac{D}{2}\right) w(w-1) .
\end{gathered}
$$

Рассмотрим подробнее уравнения спиновой динамики на примере уравнения (PVI). Лагранжиан (37) описывает динамику спина, величина которого $(\vec{S}, \vec{S})=\mu^{2}$, в кристаллическом поле $\hat{J}$ и переменном магнитном поле $\vec{h}$ :

$$
\frac{i}{2} x(x-1) \vec{S}_{x}=\left[\vec{S} \times \frac{\delta H}{\delta \vec{S}}\right], \quad H=\frac{1}{4}(\vec{S} \hat{J} \vec{S})+(\vec{h} \vec{S}),
$$

причем симметричная матрица $\hat{J}$ имеет вид

$$
\begin{gathered}
-J_{33}=1+x, \quad J_{23}=J_{32}=\frac{1}{2 i}(1+x) \\
J_{13}=J_{31}=\frac{1}{2}(1-x), \quad J_{11}=J_{22}=J_{12}=J_{21}=0,
\end{gathered}
$$

а магнитное поле -

$$
\begin{aligned}
h_{1}+i h_{2} & =\left(A+B+C+1-\frac{3}{2} \mu\right), \quad-h_{1}+i h_{2}=x\left(A-\frac{1}{2} \mu\right), \\
h_{3} & =\frac{1}{2}\left[(x+1)\left(\frac{\mu}{2}-A\right)+x\left(\frac{\mu}{2}-B\right)+\left(\frac{\mu}{2}-C-1\right)\right] .
\end{aligned}
$$

Лагранжиан (37) в новых переменных имеет вид ( $\mu=D$ или $\mu=A+B+C+1-D)$

$$
\begin{gathered}
L=w_{x} p-\frac{1}{x(x-1)}\left[\frac{1}{4}(\vec{S} \hat{J} \vec{S})+(\vec{h} \vec{S})\right] \\
\vec{S}=\vec{M} p+\frac{\mu}{2} \vec{M}_{w}, \quad \vec{M}=\left(w^{2}-1, i\left(w^{2}+1\right), 2 w\right) .
\end{gathered}
$$


Определим новую параметризацию вектора

$$
\vec{M}=\left(\frac{w^{2}-x}{\sqrt{x}}, \frac{w^{2}-2 w+x}{\sqrt{1-x}}, \frac{w^{2}-2 w x+x}{\sqrt{x(x-1)}}\right)
$$

которая получается из (40) поворотом в спиновом пространстве, зависящим от $x$, но не от $w$. При этом вид лагранжиана (40) не меняется, а анизотропия и магнитное поле имеют вид

$$
\begin{gathered}
\hat{J}=\operatorname{diag}(0,-1,-x), \\
\vec{h}=\frac{1}{2}((B+C+1-\mu) \sqrt{x},(A+C+1-\mu) \sqrt{1-x},(A+B-\mu) \sqrt{x(x-1)}) .
\end{gathered}
$$

Для того чтобы получить уравнение типа (39), отметим, что надо учесть явную зависимость $\vec{S}$ от $x$ :

$$
\frac{i}{2} x(x-1) \frac{\partial \vec{S}(p w x)}{\partial x}=\left[\vec{S} \times \vec{h}^{x}\right], \quad \vec{h}^{x}= \pm \frac{1}{4}(\sqrt{x}, \sqrt{1-x}, \sqrt{x(x-1)})
$$

Тогда уравнение спиновой динамики будет иметь вид (39), причем анизотропия определена в (41), а магнитное поле преобразуется следуюшим образом:

$$
\vec{h} \rightarrow \vec{h}+\vec{h}^{x}
$$

\section{4. ЗАКЛЮЧЕНИЕ}

В данной работе получено представление кулоновского газа для рациональных решений уравнений Пенлеве. Для уравнения Пенлеве IV можно использовать автомодельную редукцию многочастичной динамической системы (типа Калоджеро-Мозера), описывающей динамику полюсов рациональных решений системы Леви. Но такой подходне всегда удобен, поскольку динамические системы, которые редуцируются к уравнениям Пенлеве, либо очень сложны, либо переходят в уравнения Пенлеве с неполным набором параметров. Альтернативой является рассмотрение различных замыканий дискретной решетки преобразований Беклунда системы Леви [6], из которых получаются все уравнения Пенлеве. Однако наиболее удобным способом получения уравнений для полюсов является анализ поведения решений вблизи особых точек.

В работе показано, что любое рациональное решение уравнения Пенлеве определяет какую-либо стационарную конфигурацию электрических зарядов. Обратное, вообще говоря, неверно. Автор благодарен А. П. Веселову, указавшему на это обстоятельство.

Нерешенной остается задача о решениях уравнений кулоновского газа, которые являются комплексными числами. Требуется также, чтобы физическая конфигурация допускала поворот в плоскости. Потенциал взаимодействия допускает такой поворот, однако, например, для уравнения Пенлеве IV потенциал имеет вид $V=V_{0} z^{2}$, а 
не $V=V_{0} \bar{z} z$, что приводит к тому, что все полюсы рациональных решений уравнения (PIV) лежат либо на вешественной, либо на мнимой оси. Было бы интересно найти комплексное обобщение уравнения (PIV) с симметричным параболическим потенциалом (заметим, что в случае уравнения (PII) таких трудностей не возникает, так как внешний потенциал вообще отсутствует).

Представление уравнений Пенлеве в гамильтоновом виде удобно для получения спиновых моделей и для построения всех преобразований Беклунда, однако вопрос о построении полной решетки преобразований Беклунда до сих пор не решен. Трудность заключается в том, что наличие дополнительных точечных преобразований, относительно которых инвариантно соответствуюшее уравнение Пенлеве, затрудняет вопрос о ближайших соседях в решетке. В данной работе ответ на этот вопрос дан для уравнения Пенлеве IV.

Благодарности. Автор благодарен А. Б. Шабату за полезные обсуждения. Работа выполнена при частичной финансовой поддержке Российского фонда фундаментальных исследований (грант № 98-01-01161) и программы "Научные школы Российской Федерации" (грант № 96-15-96632).

\section{Список литературы}

[1] Е. Л. Айнс. Обыкновенные диффференциальные уравнения. Харьков: ДНТВУ, 1939.

[2] M. Boiti, F. Pempinelli. Nuovo Cimento B. 1980. V. 59. P. 40-58.

[3] В. Г. Марихин, А. Б. Шабат, М. Бойти, Ф. Пемпинелли. ЖЭТФ. 2000. Т. 117. № 3. C. $634-643$

[4] М. Абловиц, Х. Сигур. Солитоны и метод обратной задачи. М.: Мир, 1987.

[5] В. Г. Марихин, А. Б. Шабат. ТМФ. 1999. Т. 118. № 2. С. 217-228.

[6] В. Э. Адлер, А. Б. Шабат, Р. И. Ямилов. ТМФ. 2000. Т. 125. № 3. С. 355-424.

[7] В. Е. Захаров, С. В. Манаков, С. П. Новиков, Л. П. Питаевский. Теория солитонов. М.: Наука, 1973.

[8] K. Okamoto. Proc. Japan Acad. Ser. A. 1980. V. 56. № 6. P. 264-268.

[9] V. E. Adler. Physica D. 1994. V. 73. P. 335-351.

Поступила в редакцию 2.XI.2000 г., после доработки 4.I.2001 г. 